\title{
A feasability study of color flow doppler vectorization for automated blood flow monitoring
}

\author{
R. Schorer ${ }^{1}$ (D) A. Badoual ${ }^{2}$ - B. Bastide ${ }^{3}$ A. Vandebrouck $^{3}$. \\ M. Licker ${ }^{1} \cdot$ D. Sage ${ }^{2}$
}

Received: 27 July 2016/ Accepted: 4 November 2016

(C) Springer Science+Business Media Dordrecht 2016

\begin{abstract}
An ongoing issue in vascular medicine is the measure of the blood flow. Catheterization remains the gold standard measurement method, although non-invasive techniques are an area of intense research. We hereby present a computational method for real-time measurement of the blood flow from color flow Doppler data, with a focus on simplicity and monitoring instead of diagnostics. We then analyze the performance of a proof-of-principle software implementation. We imagined a geometrical model geared towards blood flow computation from a color flow Doppler signal, and we developed a software implementation requiring only a standard diagnostic ultrasound device. Detection performance was evaluated by computing flow and its determinants (flow speed, vessel area, and ultrasound beam angle of incidence) on purposely designed synthetic and phantom-based arterial flow simulations. Flow was appropriately detected in all cases. Errors on synthetic images ranged from nonexistent to substantial depending on experimental conditions. Mean errors on
\end{abstract}

In an effort to foster open research, we will make our code and some data freely available online at http://bigwww.epfl.ch/algorithms/ blood-flow-monitoring/.

Electronic supplementary material The online version of this article (doi:10.1007/s10877-016-9953-2) contains supplementary material, which is available to authorized users.

R. Schorer

raoul.schorer@hcuge.ch

1 Department of Anaesthesiology, Pharmacology and Intensive Care, Geneva University Hospital, 1205 Geneva, Switzerland

2 Biomedical Imaging Group, Ecole Polytechnique Fédérale de Lausanne (EPFL), 1015 Lausanne, Switzerland

3 Ecole Polytechnique Fédérale de Lausanne (EPFL), 1015 Lausanne, Switzerland measurements from our phantom flow simulation ranged from 1.2 to $40.2 \%$ for angle estimation, and from 3.2 to $25.3 \%$ for real-time flow estimation. This study is a proof of concept showing that accurate measurement can be done from automated color flow Doppler signal extraction, providing the industry the opportunity for further optimization using raw ultrasound data.

Keywords Doppler · Monitoring · Computing · Ultrasound · Automation

\section{Introduction}

Arterial blood flow measurement is an essential part of monitoring and goal-directed approaches aiming at the optimization of oxygen delivery. Current gold standard methods of blood flow estimation still rely on invasive catheterization techniques [1]. Usability is however limited by time constraints and risks of iatrogeny [2]. Doppler is a well-established alternative method of flow estimation that does however suffer from the difficulties in achieving a vessel long axis view at a reliable angle, and in getting a centrally-located and appropriately-gated sampling region [3]. Doppler therefore requires an experienced operator, while operator independence is a crucial issue in blood flow estimation [4]. Computer vision techniques have been applied as potential solutions to make Doppler manipulation simpler and more reliable [5]. We present in this work a new and fully automated color flow Doppler processing concept for blood flow monitoring. Using only widely available standard ultrasound hardware, we perform a proof-of-principle study of a software implementation on synthetic and phantom simulation-based samples. Access to raw ultrasound data instead of video samples would 
obviously yield better results, although our study does nevertheless open new perspectives on building a reasonably accurate and simple, non-invasive clinical monitor.

\section{Material and methods}

\subsection{Compliance with ethical standards}

Author A. Badoual was funded by the Swiss National Science Foundation (grant 200020-162343). No other funding supported the present study. Authors declare that they have no conflict of interest. This article does not contain any studies with human participants or animals performed by any of the authors, at the exception of a proof-of-concept demonstration performed by the first author on himself, for which no formal consent was required. No informed consent was required for this study.

\subsection{Algorithm}

Geometrical model We modeled the target blood vessel as a perfect open cylinder, and considered the oblique short axis cross-sectional profile as a projection of the orthogonal short axis profile on an oblique plane intersecting the cylinder (Fig. 1). Detected blood flow speed is subject to the same projection, therefore depending on the angle of incidence of the ultrasound beam. Flow will be the product of the projected vessel cross-sectional area and the projected blood flow speed. Applying those computations to every image in a sequence will yield volumetric flow with respect to time. The present model postulates the use of a

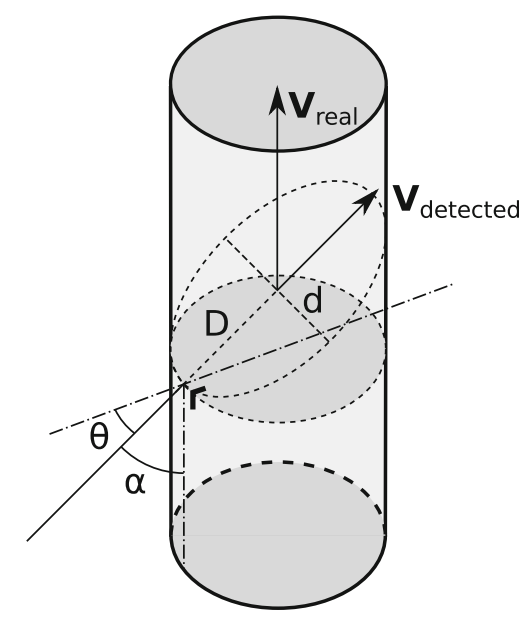

Fig. 1 A representation of our geometrical model of flow. $\theta$ : the angle of incidence, following the strict physical definition. $\alpha$ : the angle of incidence, following the medical field definition. $D$ The major axis of the ellipse defining the vessel flow cross-sectional profile (dashed). $d$ The minor axis of the ellipse. $V_{\text {real }}$ : the true axial speed of the blood. $V_{\text {detected }}$ : the detected speed of the blood linear array ultrasound probe. Adaptation to other probe types is possible, but is not described here.

Video stream processing To show that real-time monitoring could be done, we developed our own image analysis solution. Unfortunately, due to the closed system policy prevalent among diagnostic ultrasonography devices manufacturers, we had to extract relevant information from the video output of our machine (Sonosite Edge, Sonosite Inc., SE Bothwell, W.A.). This analog signal is designed by the manufacturer for PAL video format monitors $(25 \mathrm{~Hz})$. It is far from optimal as input for our analysis due to the analog conversion, interlacing, MPEG compression, and color composition. For lack of anything better, we digitalized it and used it as our source. We isolated the required information through reverse engineering. We first deinterlaced the video stream, which resulted in a fourfold increase in temporal resolution to $100 \mathrm{~Hz}$, and 16-times smaller sample images $(180 \times 144$ pixels $)$. B-mode and Doppler signals were separated through green channel subtraction. The region of interest and the color scale were then automatically detected using the Hough lines ridge detection algorithm [6]. The target vessel was isolated using a two-pass connected component algorithm. Finally, a crucial step to quantify flow speed was to remap the color scale to color-speed pairs, based on the user-defined maximal speed value. The full process can be visualized on Fig. 2, and a detailed flow chart can be found in Fig. 3.

Flow computation With the above requisites fulfilled, we computed the volumetric flow and the ultrasound beam angle of incidence. To that effect, we fitted an ellipse to the target vessel. Following our geometrical model, the ratio of the minor and major axes of the fitted ellipse defined the cosine of the ultrasound beam angle of incidence. Knowing that, we retrieved the color value of every pixel of interest and mapped it to its corresponding speed. Volumetric flow was then computed as the product of the corrected area and mean speed.

\subsection{Validation}

Synthetic images We generated interlaced and non-interlaced images similar to those yielded by our ultrasound machine. Each image included the color scale, a quadrangle defining the region of interest, and a random noise background (Fig. 4, top row). Three distinct sets of images were generated, specifically designed to respectively test our ability to detect the angle of incidence $(\alpha)$, map the blood speed to each color, and compute the vessel crosssectional area. For angle estimation tests, eighteen images were designed to represent the vessel profile that would normally be seen with angle of incidence values going from $5^{\circ}$ to $90^{\circ}$ by $5^{\circ}$ steps. For speed tests, we created 
Fig. 2 A screenshot of our software on the author's common carotid artery. The central window shows the unmodified color flow Doppler video as a still image $(A)$. Horizontal line artifacts are generated from the video interlacing. Flow and estimated angle of incidence values are displayed in the lower left corner. The flow with respect to time graph can be seen in the lower section $(B)$. Columns on the right side demonstrate the region of interest definition and deinterlacing procedures $(C)$, and the result of the segmentation process $(D)$
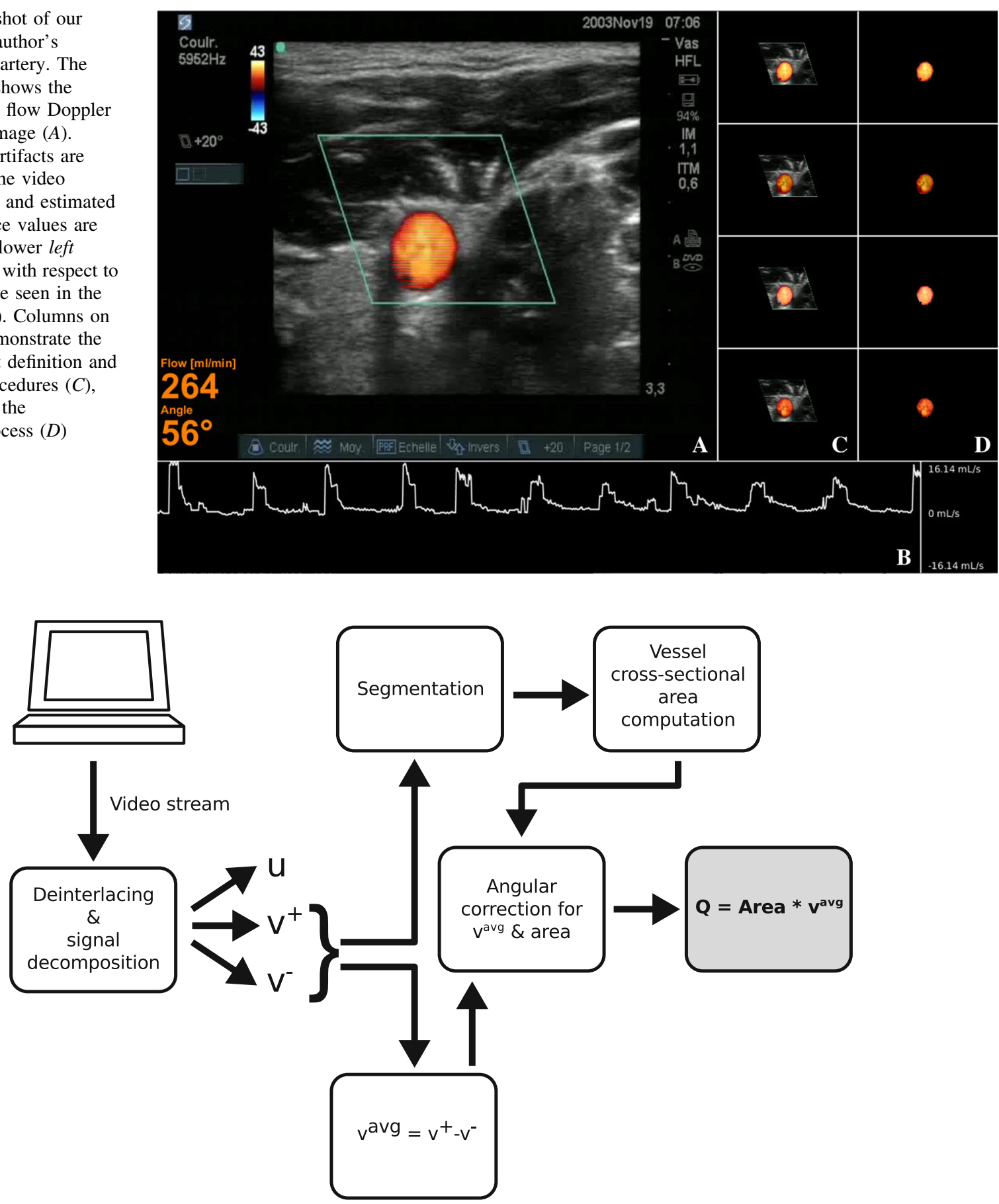

Fig. 3 Flowchart of the main stages of our software implementation. The video stream is digitalized from a standard diagnostic ultrasound device (top left). Captured frames are then deinterlaced to their four distinct fields for region of interest selection. B-mode $(u)$ and speed signals $\left(v^{+}\right.$, and $\left.v^{-}\right)$are extracted from the composite image.

nineteen images comprising a simulated vessel of a color chosen among the nineteen available colors of the scale. Finally, test data for cross-sectional area computation consisted in eighteen pairs of interlaced and non-interlaced images including a vessel profile of constant 1000 pixels
Connected components segmentation provides the vessel area, and angular correction occurs through ellipse fitting. Average speed $v^{a v g}$ is computed as the subtraction of the opposing speed signals $v^{+}$and $v^{-}$. The resulting speed and area, after angle correction, are multiplied to yield the volumetric flow value $(\mathrm{Q}$, grey node)

area, the shape of which varied to simulate angle of incidence values ranging from $5^{\circ}$ to $90^{\circ}$. This also allowed us to evaluate the impact of the interlacing process through direct comparison of detection accuracy on interlaced and non-interlaced images. 

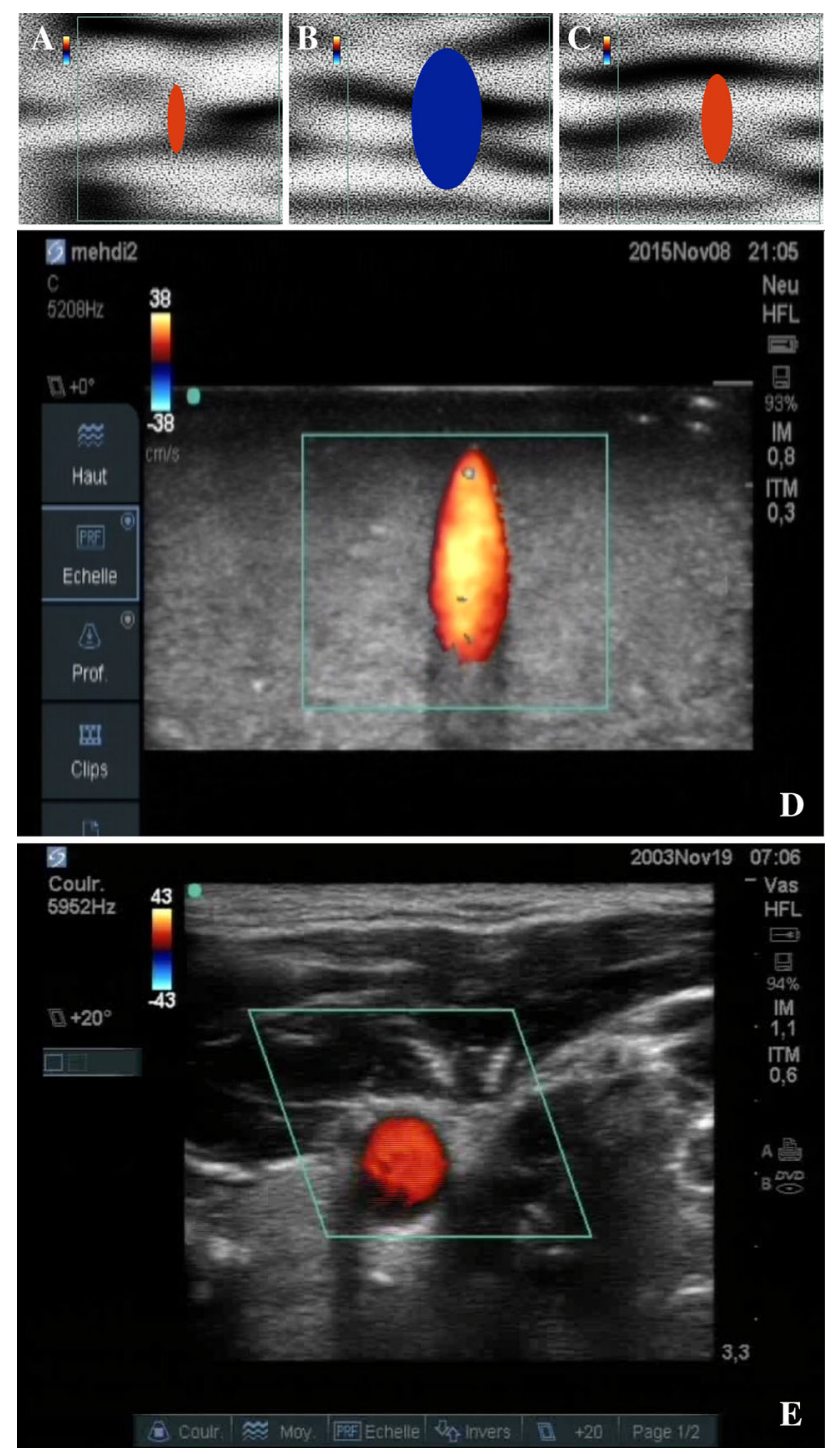

Fig. 4 A comparison of example synthetic test images for angle (a), speed (b), area detection (c), phantom images (d), and natural images (e). The green quadrangle surrounding the vessel and the colorscale displayed by our ultrasound machine were reproduced on synthetic images to enable correct analysis

Phantom experimental setting A blood flow simulation circuit was designed using a peristaltic pump (Ismatec MVGE, IDEX Health \& Science, Wertheim, Germany) running at controlled speeds, and calibrated by measuring the transferred volume of $0.75 \%$ corn starch-in-water solution from an inflow tank to a graduated outflow cylinder over one minute (Fig. 5). The pump produced a sinusoidal pulsatile flow. The site of measurement consisted in a silicone Penrose drain immersed in a $4 \%$ corn starch-in-water solution. The phantom was an open circuit with no recirculation. For each experimental condition, the circuit flow was calculated by measuring the time necessary to transfer one liter of solution to the outflow cylinder, and reproducibility was ascertained by doing it five times before beginning Doppler measurements. The repeatability error from the graduated cylinder was consistently smaller than $1 \%$, and has not been considered in our data analysis. Measurements were conducted by recording color flow Doppler videos of thirteen successive circuit runs for each experimental condition, across four incremental pump speeds, totalizing 110 minutes of video sampling. All flow measurements were made with an ultrasound beam incidence of $45^{\circ}$. Angle of incidence validation was performed following identical principles, with four one-minute replicates by $10^{\circ}$ angle increment from $20^{\circ}$ to $60^{\circ}$. Those twenty experimental samples amounted to 12 minutes of sampling. We tuned the ultrasound machine to center the mean detected flow speed in the upper third of the color scale.

\section{Results}

\subsection{Synthetic images}

Angle estimation tests Analysis of synthetic images revealed a variable performance across experimental conditions. Angle measurement errors tended to increase with angle of incidence (range $0.15-13.11 \%$ ). The error kept low in the angle range recommended for use in the medical field $\left(<60^{\circ}\right)$, with a maximum of $5.3 \%$. Results of angle estimations on synthetic images can be consulted in Table 1, and a test image example can be seen in Fig. 4.

Speed estimation tests Errors on speed ranged from low to acceptable in the mid to high range of the speed scale (minimal error $0.23 \%$ ), but logically increased in proportion in the low range, reaching $101.8 \%$. Testing the color corresponding to a speed of zero did however yield the exact value. The errors on speed (not considering direction, $\Delta V=\mid$ Expected $V|-| V \mid$ in Table 2) were confirmed as random by a non-significant single-sample t-test $(\mathrm{p}=0.39$, $95 \%$ confidence interval on the mean [CI95] -0.147 to $0.358 \mathrm{~cm} / \mathrm{s}$ ). There was however a significant overestimation trend in velocity (i.e considering direction, $\Delta V=$ Expected $V-V$, not shown) confirmed by a onesample sign test $(\mathrm{p}=0.008$, CI95 $0.017-0.499 \mathrm{~cm} / \mathrm{s})$. Results of speed estimation from synthetic images are in Table 2, and a test image example can be seen in Fig. 4.

Cross-sectional area computation tests Error on crosssectional area did not increase beyond a few percent of the expected value $(2.2-8.25 \%)$. While not following a clear linear trend, the error tended to vary in an inverse fashion when compared to the angle of incidence, with the maximal error being associated with the smallest angle value. Table 3 summarizes area detection results on synthetic images, while an example of a test image can be found in 
Fig. 5 Our experimental setting. a Inflow tank containing a $0.75 \%$ corn starch-in-water solution. b Outflow graduated cylinder allowing precise quantification of the collected liquid. c Peristaltic rotational pump generating a pulsatile flow across the circuit.

d Measurement tank containing a segment of circuit constituted by a Penrose silicone drain immersed in a $4 \%$ corn starchin-water solution. e Ultrasound probe

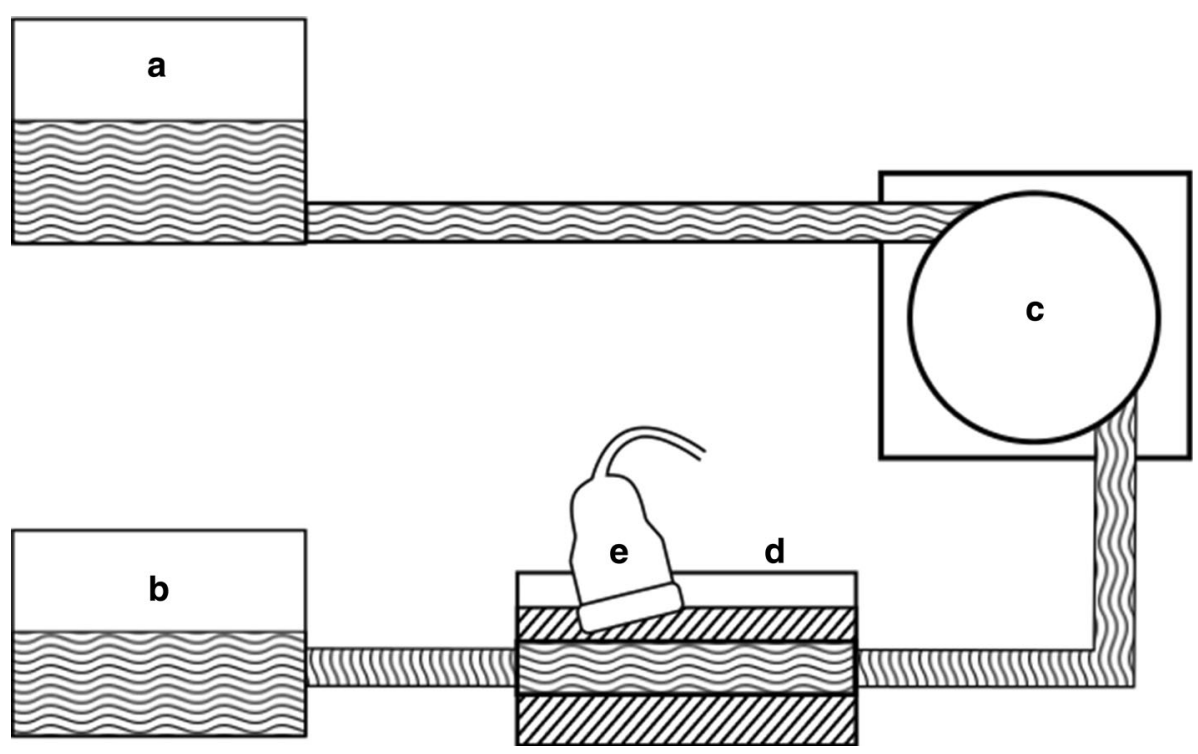

Table 1 Error summary of tests conducted on synthetic images for angle of incidence $(\alpha)$ estimation

\begin{tabular}{lccc}
\hline Expected $\alpha^{\circ}$ & $\alpha^{\circ}$ & $\Delta \alpha^{\circ}$ & $\Delta \alpha[\%]$ \\
\hline 5 & 4.952 & -0.048 & 0.95 \\
10 & 9.892 & -0.108 & 1.08 \\
15 & 14.974 & -0.026 & 0.17 \\
20 & 20.173 & 0.173 & 0.87 \\
25 & 25.036 & 0.036 & 0.15 \\
30 & 30.269 & 0.269 & 0.9 \\
35 & 35.32 & 0.32 & 0.92 \\
40 & 41.27 & 1.27 & 3.18 \\
45 & 46.61 & 1.61 & 3.58 \\
50 & 51.938 & 1.938 & 3.88 \\
55 & 57.913 & 2.913 & 5.3 \\
60 & 61.43 & 1.43 & 2.38 \\
65 & 71.007 & 6.007 & 9.24 \\
70 & 71.007 & 1.007 & 1.44 \\
75 & 76.759 & 1.759 & 2.35 \\
80 & 76.499 & -3.501 & 4.38 \\
85 & 77.017 & -7.983 & 9.39 \\
90 & 78.197 & -11.803 & 13.11 \\
\hline 7 & & &
\end{tabular}

Test images were non-interlaced

Fig. 4. Figure 6 shows that the interlacing process generally degraded the accuracy and precision of measurements, as evidenced by both a higher mean error (4.45 vs. 6.98\%) and a larger confidence interval (3.8-5.2 vs. 4.77-9.2\%).

\subsection{Phantom experimental setting}

Angle Angle measurements could be made over the medical field recommended angle range of $20^{\circ}$ to $60^{\circ}$, with
Table 2 Error summary of tests conducted on synthetic images for speed (V) estimation. Test images were non-interlaced

\begin{tabular}{|c|c|c|c|}
\hline Expected V $(\mathrm{cm} / \mathrm{s})$ & $\mathrm{V}(\mathrm{cm} / \mathrm{s})$ & $\Delta \mathrm{V}(\mathrm{cm} / \mathrm{s})$ & $\Delta \mathrm{V}(\%)$ \\
\hline 9 & 9.38 & 0.38 & 4.23 \\
\hline 8 & 8.491 & 0.491 & 6.14 \\
\hline 7 & 7.482 & 0.482 & 6.88 \\
\hline 6 & 6.502 & 0.502 & 8.37 \\
\hline 5 & 5.726 & 0.726 & 14.51 \\
\hline 4 & 4.499 & 0.499 & 12.48 \\
\hline 3 & 2.021 & -0.979 & 32.63 \\
\hline 2 & 2.34 & 0.34 & 16.98 \\
\hline 1 & 2.018 & 1.018 & 101.8 \\
\hline 0 & 0 & 0 & 0 \\
\hline-1 & -0.501 & -0.499 & -49.92 \\
\hline-2 & -2.328 & 0.328 & -16.39 \\
\hline-3 & -2.988 & -0.012 & -0.41 \\
\hline-4 & -3.503 & -0.497 & -12.42 \\
\hline-5 & -4.506 & -0.494 & -9.89 \\
\hline-6 & -5.762 & -0.238 & -3.97 \\
\hline-7 & -7.479 & 0.479 & -6.85 \\
\hline-8 & -7.981 & -0.019 & -0.23 \\
\hline-9 & -8.496 & -0.504 & -5.6 \\
\hline
\end{tabular}

$\overline{\Delta V \text { is defined as } \mid \text { Expected } V|-| V \mid \text { and therefore does not consider }}$ direction

errors ranging from $1.25 \%$ (CI95 $1.2-1.3 \%$ ) to $40.23 \%$ (CI95 40.13-40.33\%). Measurements at incidence values of less than $20^{\circ}$ could not be performed due to the configuration of our flow phantom. The percent error was greatest at an incidence of $20^{\circ}$, as anticipated. Our data showed strong interobservation agreement based on the intraclass correlation coefficient (ICC 0.72, CI95 
Table 3 Error summary of tests conducted on synthetic images for vessel cross-sectional area estimation at various angles of incidence $(\alpha)$

\begin{tabular}{llll}
\hline$\alpha^{\circ}$ & Area (pixels) & $\Delta$ Area (pixels) & $\Delta$ Area $(\%)$ \\
\hline 5 & 1082 & 82 & 8.2 \\
10 & 1067 & 67 & 6.7 \\
15 & 1044 & 44 & 4.4 \\
20 & 1044 & 44 & 4.4 \\
25 & 1035 & 35 & 3.5 \\
30 & 1034 & 34 & 3.4 \\
35 & 1031 & 31 & 3.1 \\
40 & 1034 & 34 & 3.4 \\
45 & 1042 & 42 & 4.2 \\
50 & 1052 & 52 & 5.2 \\
55 & 1043 & 43 & 4.3 \\
60 & 1048 & 48 & 4.8 \\
65 & 1039 & 39 & 3.9 \\
70 & 1052 & 52 & 5.2 \\
75 & 1053 & 53 & 5.3 \\
80 & 1035 & 35 & 3.5 \\
85 & 1053 & 53 & 5.3 \\
90 & 1022 & 22 & 2.2 \\
\hline
\end{tabular}

Test images were non-interlaced

0.32-0.96). Results from angle estimation on phantom measurements may be consulted in Table 4.

Flow The detected flow ranged from $0.237 \mathrm{1} / \mathrm{min}$ to $2.388 \mathrm{l} / \mathrm{min}$, with errors ranging from $3.17 \%$ (CI95 1.14$5.2 \%$ ) to $-25.27 \%$ (CI95 -27.12 to $-23.43 \%$ ). The lowest error was found in the $0.23 \mathrm{l} / \mathrm{min}$ experimental condition, and the highest in the $0.82 \mathrm{l} / \mathrm{min}$ condition. Moderate interobservation agreement based on intraclass correlation was found (ICC 0.54, CI95 0.22-0.95). Results from our phantom flow assays are summarized in Table 5.

\section{Discussion}

The main point in our results is that the color flow Doppler vectorization process behaved as theoretically expected from our geometrical model in almost all aspects, suggesting a potential for the development of software-based automated signal processing applications in ultrasonography using computer vision.

\subsection{Comparison to prior work}

To the best of our knowledge, all attempts to apply computer vision to Doppler signals were based on either spectral or long axis color flow Doppler, this work being the first to apply it to color flow Doppler using the short

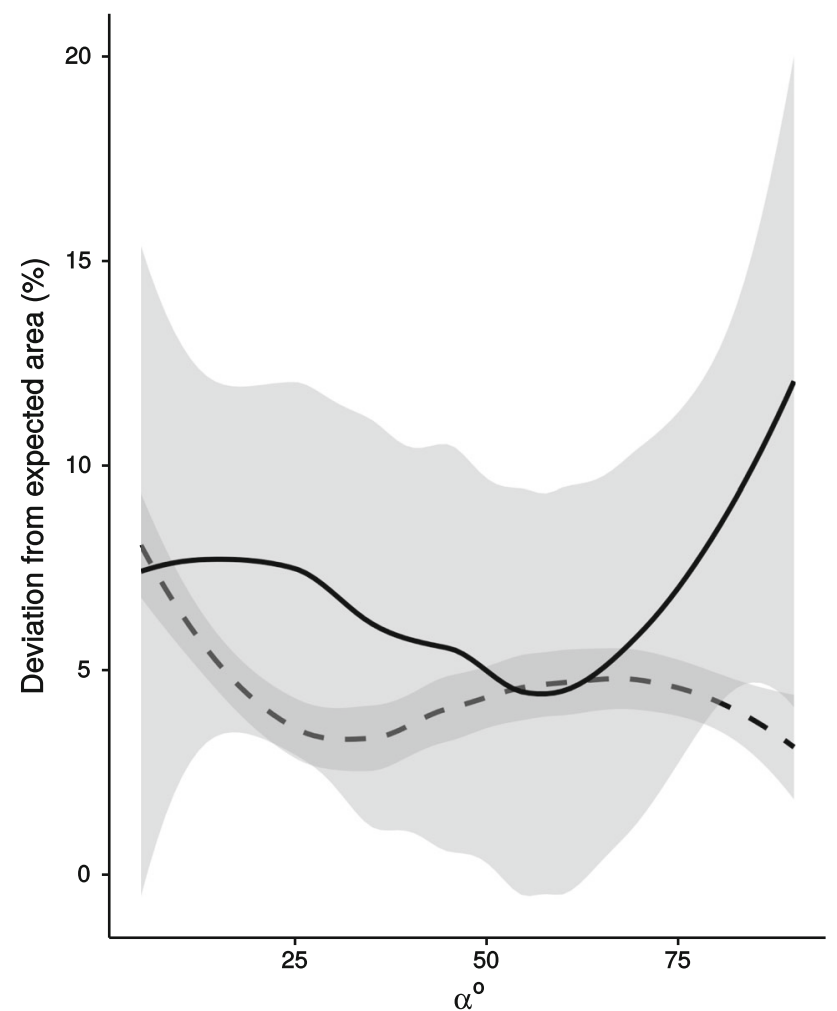

Fig. 6 Smoothed line plot of the impact of interlacing four subfields of size 1/16th (solid line, four $180 \times 144$ pixels fields, $720 \times 576$ pixels image) on the accuracy of vessel cross-sectional area detection, compared to a similar measurement on non-interlaced images of size 1/1 (dashed line, $720 \times 576$ pixels). Ninety-five percent confidence intervals are displayed as grey areas surrounding each line

axis vessel profile [5, 7]. However, mathematical models of Doppler flow estimation through normal vector computation have been explored in the past [8]. Compared to existing technology, one obvious advantage of our method is the ability to capture the whole flow profile of a vessel, instead of only a local sample. This allows us to handle complex flow profiles with little computational overhead. A second advantage is that keeping the probe correctly positioned in the short axis is much easier in comparison to sustaining a long axis view, favoring stable measurements. This, in addition to our built-in automated targeting process, would make our system easily accessible. Another strong point is the requirement for only a standard ultrasound probe. Indeed, many ultrasound technologies claiming angle independence rely on special probes allowing multiple sampling sites or steerable beams $[9,10]$. In the range of incidence values considered suitable for examination in the medical field $\left(<60^{\circ}\right)$, our implementation was competitive with published data on spectral Doppler techniques, but not with more modern techniques, such as vector velocity Doppler [11, 12]. An optimized implementation might improve that. 
Table 4 Results of angle of incidence $(\alpha)$ automated estimation on our blood flow simulation phantom compared to values expected by experimental design $(n=4)$

Table 5 Results of flow measurements on our blood flow simulation phantom compared to a true value based on a graduated cylinder $(n=13)$

\begin{tabular}{llccll}
\hline True angle $\left(^{\circ}\right)$ & $\alpha^{\circ}$ & $\Delta \alpha^{\circ}$ & $\Delta \alpha[\%]$ & $\Delta \alpha^{\circ}$ CI95 & $\Delta \alpha$ CI95[\%] \\
\hline 20 & 28.047 & 8.047 & 40.23 & $8.027-8.067$ & $40.13-40.33$ \\
30 & 30.857 & 0.857 & 2.86 & $0.816-0.898$ & $2.72-2.3$ \\
40 & 42.761 & 2.761 & 6.9 & $2.693-2.829$ & $6.73-7.07$ \\
50 & 50.623 & 0.623 & 1.25 & $0.598-0.648$ & $1.2-1.3$ \\
60 & 55.355 & -4.645 & -7.74 & -4.711 to -4.579 & -7.85 to -7.63 \\
\hline
\end{tabular}

\begin{tabular}{llcrll}
\hline True flow $(1 / \mathrm{min})$ & $\dot{Q}(1 / \mathrm{min})$ & $\Delta \dot{Q}(1 / \mathrm{min})$ & $\Delta \dot{Q}(\%)$ & \multicolumn{1}{l}{$\Delta \dot{Q}$ CI95 (1/min) } & \multicolumn{1}{c}{$\Delta \dot{Q}$ CI95 (\%) } \\
\hline 0.23 & 0.237 & 0.007 & 3.17 & $0.003-0.012$ & $1.14-5.2$ \\
0.56 & 0.49 & -0.07 & -12.44 & -0.078 to -0.061 & -13.99 to -10.89 \\
0.82 & 0.613 & -0.207 & -25.27 & -0.222 to -0.192 & -27.12 to -23.43 \\
2.31 & 2.388 & 0.078 & 3.37 & -0.058 to 0.214 & -2.52 to 9.23 \\
\hline
\end{tabular}

\subsection{Clinical perspectives}

Since our concept uses a cylindrical model, it is only aimed at high pressure vessels, namely arteries, and in some cases great veins. A noncircular vessel lumen, such as in atherosclerosis, will degrade the performance of our method. Our current implementation is only for linear array ultrasound probes, since other types of probe require an adaptation of the angle correction method accounting for the dispersion of the ultrasound beam.

\subsection{Technical issues}

The closed firmware policy of diagnostic ultrasound systems forced us to use video data instead of raw ultrasound data. This negatively impacted our results in many ways. We had no precise knowledge of how images were generated, and while able to reverse-engineer B-mode and color flow Doppler signals, we still ended with images representing an already thresholded signal. Other information making calibration unreliable, such as the potential non-linearity of the color scale speed mapping, is ignored and difficult to test experimentally. Accessing the original signal would allow us to implement an accurate and robust image processing algorithm without thresholding, such as modern segmentation techniques like active contours $[13,14]$. In addition, the need for color scale speed remapping would obviously be unnecessary. Although it is not possible to foresee how such a system would compare to other flow computation methods, a software approach still offers numerous advantages over a hardware implementation: ease of update, and richer signal postprocessing such as shape constraint, robustness to perturbations, or complex targeting algorithms. The operator would be spared the manual operations traditionally required when estimating flow. Also noteworthy, the absence of heavy computation requirements avoids degrading the time and space sampling capabilities of the ultrasound machine.

\subsection{Experimental data}

Synthetic data Tests performed on synthetic images revealed some amount of error. Image aliasing and the use of a fixed threshold made region of interest selection slightly generous (Table 3 ). The increasing speed percent error in the low range of the scale reflected this. Since the shape of a vessel will vary only slightly across the high ranges of angle of incidence, the incorrectly detected pixels also held a far greater influence at those ranges. A general trend of increasing angle measurement errors can indeed be seen with increasing incidence values (Table 1). We concentrated on speed estimation (disregarding direction) instead of velocity, since our priority was to ascertain the reliability of our method (Table 2). In that regard, the small systematic error on velocity (considering direction) is less concerning, since imperfect precision can safely be assumed to be due to technical limitations. Specifically, potential sources of error are the use of a fixed threshold in our image pipeline, and miscalibration of the ultrasound hardware. Both sources are therefore correctable by an improved implementation, and cannot be linked to the theoretical aspect of our model.

The necessity of image deinterlacing to subfields of surface 1/16th implied a spatial resolution decreased by a factor of 16 compared to equivalent non-interlaced images, although this was somewhat mitigated by averaging. Our tests on phantom-simulated images suffered this penalty, as can be well understood by looking at Fig. 6 .

Phantom data The interobservation agreement levels exhibited by our data are encouraging, since they likely reflect a systematic bias that might be reduced through an optimized implementation. It was expected that 
measurement inaccuracies would increase along with speed, based on the fact that the color scale included only nineteen distinct colors, meaning that assuming a linear speed-to-color mapping, the uncertainty on measurement was theoretically equal to $\pm\left|V_{\max }\right| / 18$, where $V_{\max }$ was the speed scale maximum. By looking at Table 5, one can see that absolute confidence intervals (column $\Delta \dot{Q}$ CI95 [1/ min]) seemed to follow that trend, although such was not the case for the mean bias on measurement (column $\Delta \dot{Q}[1 /$ $\mathrm{min}]$ ). Other confounding factors likely play a role.

Phantom angle estimation data was similarly encouraging. Absolute errors of angle estimation did not go beyond a few degrees (Table 4). Secondly, we expected increasing confidence intervals along with angle of incidence values, since a given error held a proportionally greater importance at high ranges of incidence. This was however not the case in the tested angle range.

Finally, within-condition measurements appeared quite stable with respect to time, as evidenced by relatively narrow confidence intervals in both angle and flow estimates (Tables 4, 5). This suggests satisfactory beat-to-beat reproducibility on a stable pulsatile signal such as that produced by our pump.

\subsection{Study limitations}

The present results do not fully reflect the performance of our concept, and we cannot estimate how much better a machine with our method built in will be. Technical constraints with the phantom simulation did not allow us to explore a potential relationship between ultrasound beam angle of incidence and detected flow rate. This implies that although suggested by the concept, we cannot yet formally claim angle independence. Finally, we performed preliminary tests of our implementation on natural images, but the transfer of our concept to live subject data remains to be studied and we will not present it here (Fig. 2).

\subsection{Future work}

Complex targeting A custom targeting criterion could be easily implemented, since it is distinct from the flow computation algorithm. Our system would allow any level of complexity in the target selection, including multiple vessels, selection on flow direction, selection on the pulsatility profile, etc. For example, the ability to select multiple targets would enable measurements in a whole vascular bed, such as the arcuate arteries of the kidney, allowing the user to differentiate cortical and medullary kidney perfusion. Complex targeting is therefore not only a matter of ease of use, but could also lead to interesting clinical applications.
Improved segmentation The acquisition of the raw Doppler signal would allow the use of state-of-the-art segmentation techniques, such as active contours (a.k.a. snakes) $[13,14]$. This could also allow better analysis of the time-dependent signal variations, which would be a perfect use case for a snake segmenting in both time and space dimensions. From a practical point of view, this would also mean enhanced automated adjustment of the image settings for the user, instead of the currently used manual tuning.

Continuous monitoring If future research shows consistent results on natural images, continuous monitoring will be a logical application of our method. We therefore plan to use dedicated probes, including the transesophageal modality, that could be attached to the experimental subject for longer term measurements. This would also allow the quantification of the minimal energy transfer necessary for a satisfactory measurement.

\section{Conclusion}

Results suggest that short axis color flow Doppler analysis may allow accurate blood flow measurements. Based on this proof-of-principle study, we think this might represent an opportunity for the industry to refine and improve the process, hopefully leading to substantially improved results.

Acknowledgements This work used $\mathrm{R}$ and the following $\mathrm{R}$ packages for data analysis: knitr, xtable, ggplot2, plyr, dplyr [15-20].

Funding Author A. Badoual was funded by the Swiss National Science Foundation under grant 200020-162343.

\section{Compliance with ethical standards}

Conflict of interest The authors declare that they have no conflict of interest.

\section{References}

1. Barnett CF, Vaduganathan M, Lan G, Butler J, Gheorghiade M. Critical reappraisal of pulmonary artery catheterization and invasive hemodynamic assessment in acute heart failure. Expert Rev Cardiovasc Ther. 2013;11(4):417-24. doi:10.1586/erc.13.28.

2. Nellaiyappan M, Omar HR, Justiz R, Sprenker C, Camporesi EM, Mangar D. Pulmonary artery pseudoaneurysm after Swan-Ganz catheterization: a case presentation and review of literature. Eur Heart J Acute Cardiovasc Care. 2014;3(3):281-8. doi:10.1177/ 2048872613520252.

3. Steinman AH, Tavakkoli J, Myers JGJ, Cobbold RS, Johnston KW. Sources of error in maximum velocity estimation using linear phased-array Doppler systems with steady flow. Ultrasound Med Biol. 2001;27(5):655-64. 
4. Mead GE, Lewis SC, Wardlaw JM. Variability in Doppler ultrasound influences referral of patients for carotid surgery. Eur $\mathbf{J}$ Ultrasound. 2000;12(2):137-43.

5. Saad AA, Loupas T, Shapiro LG. Computer vision approach for ultrasound Doppler angle estimation. J Digit Imaging. 2009;22(6):681-8. doi:10.1007/s10278-008-9131-2.

6. Duda RO, Hart PE. Use of the Hough transformation to detect lines and curves in pictures. Commun ACM. 1972;15(1):11-5. doi: $10.1145 / 361237.361242$.

7. Hirsch A, Petersch B, Honigmann D. Automated Doppler gate placement and velocity calculation based on a vessel angle estimate. Conf Proc IEEE Eng Med Biol Soc. 2006;1:4461-4. doi:10. 1109/IEMBS.2006.260121.

8. Kim WY, Poulsen JK, Terp K, Staalsen NH. A new Doppler method for quantification of volumetric flow: in vivo validation using color Doppler. J Am Coll Cardiol. 1996;27(1):182-92. doi:10.1016/0735-1097(95)00435-1.

9. Dunmire B, Beach K, Labs KH, Plett M, Strandness D Jr. Crossbeam vector Doppler ultrasound for angle-independent velocity measurements. Ultrasound Med Biol. 26(8):1213-35. doi:10. 1016/S0301-5629(00)00287-8.

10. Tortoli P, Dallai A, Boni E, Francalanci L, Ricci S, An Automatic Angle Tracking Procedure for Feasible Vector Doppler Blood Velocity Measurements. Ultrasound Med Biol. 36(3):488-496. doi:10.1016/j.ultrasmedbio.2009.11.004.

11. Ho SS, Metreweli C. Preferred technique for blood flow volume measurement in cerebrovascular disease. Stroke. 2000;31(6):1342-5.
12. Tortoli P, Lenge M, Righi D, Ciuti G, Liebgott H, Ricci S. Comparison of carotid artery blood velocity measurements by vector and standard Doppler approaches. Ultrasound Med Biol. 2015;41(5):1354-62. doi:10.1016/j.ultrasmedbio.2015.01.008.

13. Jacob M, Blu T, Unser M. Efficient energies and algorithms for parametric snakes. IEEE Trans Image Process. 2004;13(9):1231-44.

14. Delgado-Gonzalo R, Uhlmann V, Schmitter D, Unser M. Snakes on a plane: a perfect snap for bioimage analysis. IEEE Signal Process Mag. 2015;32(1):41-8.

15. R Core Team: R, A language and environment for statistical computing. R Foundation for Statistical Computing, Vienna, Austria. 2015. https://www.R-project.org/

16. Xie Y. knitr: a general-purpose package for dynamic report generation in R; 2016. http://yihui.name/knitr/. R package version 1.13

17. Dahl DB. xtable: Export Tables to LaTeX or HTML; 2016. https://R-Forge.R-project.org/projects/xtable/. R package version $1.8-3 / \mathrm{r} 112$

18. Wickham H. ggplot2: elegant graphics for data analysis. New York: Springer-Verlag ; 2009. http://had.co.nz/ggplot2/book

19. Wickham H. The split-apply-combine strategy for data analysis. J Stat Softw. 2011;40(1):1-29.http://www.jstatsoft.org/v40/i01/

20. Wickham H, Francois R. dplyr: a grammar of data manipulation; 2015. https://CRAN.R-project.org/package $=$ dplyr. R package version 0.4 .3 